\title{
Congenital lesions of lung in children; a diagnostic dilemma
}

\author{
Amit Singh ${ }^{1}$ \\ ${ }^{1}$ Sanjay Gandhi Post Graduate Institute of Medical Sciences
}

May 29, 2020

\begin{abstract}
Background Congenital lung anomalies pose a challenge for not only the pediatric surgeons but also for the treating physicians. Controversy exists regarding the diagnosis and the management of the cases. Objective: The aim of this study was to highlight the difficulty in diagnosis of congenital lesions of lung in children, and to create awareness among the physicians regarding avoiding the use of antitubercular treatment (ATT) and intercostal drainage (ICD) in managing these surgical conditions. Methodology: Retrospective analysis of records of all the children diagnosed with congenital lesion of lung and treated in our department from January 2005 to December 2018. Informed consent from the parents taken at the time of admission. Inclusion criteria included cases which received either ATT or ICD before being referred to our institute. Investigations include X -ray, and Contrast enhanced tomography (CECT) chest. Results: A total of 27 cases of congenital lesions of lung were operated between Jan 2005 to Dec 2018. Out of these 27, 13 (48.1\%) received either ATT or ICD and formed our study group. Out of these $13,8(61.5 \%)$ received ATT, while $5(38.5 \%)$ received ICD as initial management before being referred to us. There were 19 (70.3\%) male and $8(29.7 \%)$ females. The most common lesion was CCAM seen in 6 (46.1\%) followed by CLE $4(30.7 \%)$, BPS in $2(15.3 \%)$ and Bronchogenic cyst 1(7.6\%). Conclusion: cystic lesions of lung in children should be investigated thoroughly and ATT should not be started empirically. Pneumothorax should be differentiated from other emphysematous/ cystic lesions before submitting the child for intercostals drainage. Keywords: Cystic lesions of lung, anti-tubercular, pneumothorax, emphysema, intercostal drainage.
\end{abstract}

\section{Background}

Congenital lung anomalies pose a challenge for not only the pediatric surgeons but also for the treating physicians. Controversy exists regarding the diagnosis and the management of the cases.

Objective : The aim of this study was to highlight the difficulty in diagnosis of congenital lesions of lung in children, and to create awareness among the physicians regarding avoiding the use of antitubercular treatment (ATT) and intercostal drainage (ICD) in managing these surgical conditions.

Methodology : Retrospective analysis of records of all the children diagnosed with congenital lesion of lung and treated in our department from January 2005 to December 2018. Informed consent from the parents taken at the time of admission. Inclusion criteria included cases which received either ATT or ICD before being referred to our institute. Investigations include X -ray, and Contrast enhanced tomography (CECT) chest.

Results: A total of 27 cases of congenital lesions of lung were operated between Jan 2005 to Dec 2018. Out of these 27, 13 (48.1\%) received either ATT or ICD and formed our study group. Out of these 13, 8(61.5\%) received ATT, while $5(38.5 \%)$ received ICD as initial management before being referred to us. There were $19(70.3 \%)$ male and $8(29.7 \%)$ females.

The most common lesion was CCAM seen in $6(46.1 \%)$ followed by CLE 4(30.7\%), BPS in $2(15.3 \%)$ and Bronchogenic cyst 1(7.6\%). 
Conclusion: cystic lesions of lung in children should be investigated thoroughly and ATT should not be started empirically. Pneumothorax should be differentiated from other emphysematous/ cystic lesions before submitting the child for intercostals drainage.

Keywords : Cystic lesions of lung, anti-tubercular, pneumothorax, emphysema, intercostal drainage.

\section{Introduction}

Diagnostic dilemma occurs in cases of congenital lesions of lung in children simply because of wide range of spectrum which includes Congenital Cystic Adenomatoid Malformation (CCAM), Congenital Lobar Emphysema (CLE), Bronchopulmonary Sequestration (BPS) and Bronchogenic Cyst (BC). CCAM occurs with an incidence of 1 in 10,000 to 25,000 pregnancies, and is the commonest lung cyst. ${ }^{1}$ Children with congenital lung lesions may present at any age in life, from the newborn period to adulthood, and some of them may remain asymptomatic throughout life. ${ }^{2,3}$ Symptomatic lesions require surgical resection. ${ }^{4}$ The management of asymptomatic lesion is controversial, while some advocate surgical excision, others recommend resection only when symptoms occur. ${ }^{5,6}$ The study was undertaken to highlight the difficulties in diagnosis as many of them present to us with a misdiagnosis of either pulmonary tuberculosis or pneumothorax. By the time they present to us most of them have already received anti tubercular drugs or intercostals drainage which further complicate the management of such children suffering from cystic lesions of lung.

The aim of this study was to highlight the difficulty in diagnosis of congenital lesions of lung in children, and to create awareness among the physicians regarding avoiding the use of anti-tubercular treatment (ATT) and intercostal drainage (ICD) in managing these surgical conditions.

Methodology: Retrospective analysis of records of all the children diagnosed with congenital lesion of lung and treated in our department from January 2005 to December 2018. Informed consent from the parents taken at the time of admission.

Inclusion criteria included cases which received either ATT or intercostals drainage before being referred to us. Investigations included X -ray, Contrast enhanced tomography. All cases were operated after confirming the diagnosis by single experienced surgeon by Posterolateral thoracotomy. Resected specimens were sent for histopathological examination. Postoperatively children were followed up 06 monthly for first year then annually thereafter.

Results: A total of 27 cases of congenital lesions of lung were operated between Jan 2005 -Dec 2018. Out of these 27,13 (48.1\%) received either ATT or ICD and formed our study group. Out of these $13,8(61.5 \%)$ received ATT, while $5(38.5 \%)$ received ICD as initial management before being referred to us. There were $19(70.3 \%)$ male and $8(29.7 \%)$ females.

The most common lesion was CCAM seen in 6 (46.1\%) followed by CLE 4(30.7\%), BPS in $2(15.3 \%)$ and Bronchogenic cyst $1(7.6 \%)$. Children demographic profile, clinical presentation, site of lesions and surgical procedure done are shown in table1.

\section{Discussion:}

Congenital lesion of the lung in children is uncommon but potentially life-threatening, and warrants an urgent diagnostic work-up. ${ }^{7,8,9,10}$ These lesions may involve the lung parenchyma, bronchi, arterial supply, and venous drainage. They may present with respiratory symptoms at birth or may be detected incidentally either before or after birth. These lesions show close relationship in terms of embryology and clinical presentation. Despite the availability of the much-improved diagnostic modality the awareness of these anomalies among pediatricians is still lacking. The main reason behind the misdiagnosis of these cases is provisional diagnosis of Tuberculosis based on respiratory symptoms and a lesion on X-ray chest. In the parts of the world where Tuberculosis forms a prominent differential diagnosis of all chronic chest conditions, labelling these congenital lesions as tuberculosis is not surprising. These children are empirically prescribed ATT and in case of no response to ATT, the children are referred to higher centre for further management and hence these cases land up with us. Some cases of CLE and macro cystic variety of CCAM are misdiagnosed as 
pneumothorax and ICD insertion is done. But physicians soon realize that they are dealing with something else and they refer these cases, which form a major chunk of our study group. These mistakes do take place because of lack of awareness on part of physicians who do not suspect these conditions and hence do not look for them. Evidence for starting ATT or putting ICD is based on clinical examination, history and Chest X ray findings only. Clinical profile and Chest $\mathrm{X}$ ray are not enough to diagnose these lesions because of significant overlap of symptoms and X ray features. The gold standard for diagnosis of congenital lesion of lung in children is contrast enhanced computed tomography (CECT) chest. CCAMs presents as cystic or solid lung lesions confined to a part of lung only. They account for $25 \%$ of total congenital lesions and most commonly present with respiratory distress in newborns. Most of these lesions are now routinely diagnosed on prenatal ultrsonography. ${ }^{11,12,13,14}$ CCAM probably results from a cessation of bronchial maturation and concomitant overgrowth of mesenchymal elements, which produce the adenomatoid appearance of the anomaly in the early stage of development. ${ }^{15}$ CCAMs communicate with the bronchial tree at birth and therefore typically contain air soon after birth. ${ }^{13}$ The imaging appearance is determined by the size and number of cysts (figure 1). Lesions are typically solitary with no lobar predilection. Because of the risks of recurrent infection and malignant potential, resection remains the current treatment. ${ }^{13,14}$ Congenital lobar emphysema (CLE) is a marked pulmonary hyperinflation state that resembles all of the clinical features of obstructive emphysema. ${ }^{7,10,16,17}$ Congenital lobar emphysema usually involves a single lobe and presents with respiratory distress in the neonate. ${ }^{13,18}$ Findings of air trapping with lobar hyperinflation, mediastinal shift away from the involved lung, and compression of the ipsilateral and contralateral lung. CT will show an expanded lobe with attenuated vascular structures (figure2). It is necessary for pediatricians to evaluate associated anomalies because $14 \%$ of the cases of CLE have coexistent congenital heart disease. ${ }^{19}$ Bronchopulmonary sequestrations (BPSs) make up10-30\% of congenital cystic lung lesions. Pulmonary sequestration develops from abnormal budding of foregut and is characterized by non-functioning lung parenchyma that does not communicates with the tracheobronchial tree and have an anomalous systemic arterial supply. Bronchogenic cysts are the most common cystic lesion of the mediastinum. They usually present with recurrent infection or airway compression leading to wheezing, atelectasis, and air trapping and often present later in childhood than other congenital lung lesions. Detailed $x$ ray and CECT chest findings of common congenital lesions of lung in children are shown in table 2.

In our study group $13(48.1 \%)$ cases were misdiagnosed by primary care providers and were unnecessarily subjected to either ATT or ICD which resulted in higher morbidity in these cases either due to delay in diagnosis and management or because of the treatment per se that is ICD which complicates the surgery. Point we would like to highlight by this study is that sooner or later all congenital lesions of lung will need surgical intervention whether symptomatic or not. Therefore, they need to be diagnosed correctly before starting any treatment whether ATT or ICD. Chest X ray is not sufficient enough for making a diagnosis, CECT chest should be done in all cases of congenital lesions of lung irrespective of the age and symptoms of presentation. Although the treatment of cystic lung lesions is quite straight forward, a strong index of suspicion is essential in diagnosing this uncommon entity. This entails prompt referral to a pediatric surgical centre thereby obviating unnecessary prolonged and needless medical management.

The author would like to declare that there is no conflicts of interest and this research received no specific grant from any funding agency in the public, commercial or not-for-profit sectors.

\section{References}

1. Laberge JM, Flageole H, Pugash D, Khalife S, Blair G, Filiatrault D, et al. Outcome of the prenatally diagnosed congenital cystic adenomatoid formation: a Canadian experience. Fetal Diagn Ther 2001;16:178-86.

2. Evrard V, Ceulemans J, Coosemans W, De Baere T, De Leyn P, Deneffe G, et al Congenital parenchymatous malformations of the lung. World J Surg 2009; 23:1123-32.

3. Laberge JM, Puligandla P, Flageole H. Asymptomatic congenital lung malformations. Semin Pediatr Surg 2005;14:16-33.

4. van Leeuwen K, Teitelbaum DH, Hirschl RB, Austin E, Adelman SH, Polley TZ, et al. Prenatal diagno- 
sis of congenital cystic adenomatoid malformation and its postnatal presentation, surgical indications, and natural history. J Pediatr Surg 1999;34:794-98.

5. Calvert JK, Boyd PA, Chamberlain PC, Syed S, Lakhoo K. Outcome of antenatally suspected congenital cystic adenomatoid malformation of the lung: 10 years' experience 1991-2001.Arch Dis Child Fetal Neonatal Ed 2005

6. Aziz D, Langer JC, Tuuha SE, Ryan G, Ein SH, Kim PC. Perinatally diagnosed asymptomatic congenital cystic adenomatoid malformation: to resect or not. J Pediatr Surg 2004;39:329-34.

7. Haller JA Jr, Golladay ES, Pickard LR, Tepas JJ, Shorter NA, Shermeta DW. Surgical management of lung bud anomalies: lobar emphysema, bronchogenic cyst, cystic adenomatoid malformation, and intralobar pulmonary sequestration. Ann Thorac Surg 1979;28: 33-44.

8. Wesley JR, Heidelberger KP, DiPietro MA, Cho KJ, Coran AG. Diagnosis and management of congenital cystic disease of the lung in children. J Pediatr Surg 1986;21:202-07.

9. Schneider JR, St Cyr JA, Thompson TR, Johnson DE, Burke BA, Foker JE. The changing spectrum of cystic pulmonary lesions requiring surgical resection in infants. J Thorac Cardiovasc Surg 1985;89:33239.

10. Bailey PV, Tracy T Jr, Connors RH, deMello D, Lewis JE, Weber TR. Congenital bronchopulmonary malformations. Diagnostic and therapeutic considerations. J Thorac Cardiovasc Surg 1990;99:597-03.

11. Lucaya J, Strife J. Pediatric chest imaging: chest imaging in infants and children. eds. Berlin, Germany: Springer-Verlag, 2002:93-112.

12. Zylak CJ, Eyler WR, Spizarny DL, Stone CH. Developmental lung anomalies in the adult: radiologicpathologic radiologic pathologic correlation. RadioGraphics 2002;22:25-43.

13. Donnelly LF, Frush DP. Localized radiolucent chest lesions in neonates: causes and differentiation. AJR 1999;172:1651-58.

14. Restrepo S, Villamil MA, Rojas IC, Lemos DF, Echeverri S, Triana G, et al. Association of two respiratory congenital anomalies: tracheal diverticulum and cystic adenomatoid malformation of the lung. Pediatr Radiol2004;34:263-66.

15. Haddon MJ, Bowen A. Bronchopulmonary and neurenteric forms of foregut anomalies. Imaging for diagnosis and management. Radiol Clin North Am 1991;29:241-54.

16. Michelson E. Clinical spectrum of infantile lobar emphysema. Ann Thorac Surg 1997;24:182-96.

17. Hislop A, Reid L. New pathological findings in emphysema of childhood: poly alveolar lobar emphysema. Thorax 1970;25:682-90.

18. Tander B, Yalcin M, Yilmaz B, Ali Karadag C, Bulut M. Congenital lobar emphysema: a clinicopathologic evaluation of 14 cases. Eur J Pediatr Surg 2003;13:108-11.

19. Murray CF. Collective review. Congenital lobar emphysema. Surg Gynecol Obstet 1967;124:611-25.

Tables and figures legends

Table 1: Shows patient demographic profile, symptoms, site of lesion, diagnosis and treatment outcome.

Table 2: Shows congenital lesions of lung and their characteristic $\mathrm{x}$ ray and CECT chest findings.

Figure 1: Chest $\mathrm{x}$ ray showing lesion in right middle lobe suggestive of CCAM

Figure 2: CT chest with features suggestive of CCAM lesion. 

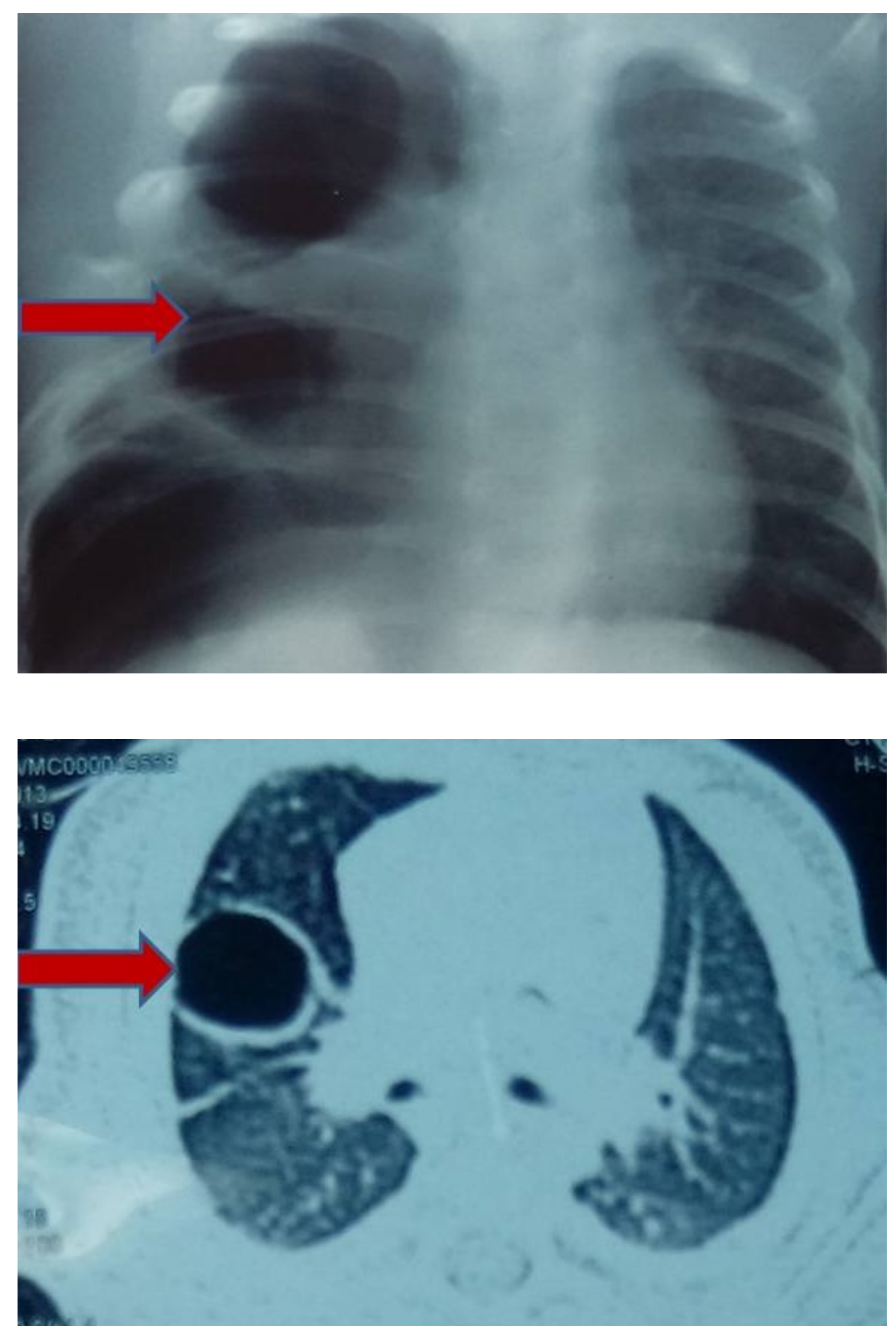

\section{Hosted file}

Table 1.doc available at https://authorea.com/users/327749/articles/455188-congenitallesions-of-lung-in-children-a-diagnostic-dilemma

\section{Hosted file}

Table 2.doc available at https://authorea.com/users/327749/articles/455188-congenitallesions-of-lung-in-children-a-diagnostic-dilemma 